J. Lake Sci. (湖泊科学), 2016, 28(6): 1283-1292

DOI 10. 18307/2016. 0614

(c) 2016 by Journal of Lake Sciences

\title{
长江中下游典型湿地沉积物-水界面硝酸盐异养还原过程
}

\author{
姜星宇 ${ }^{1,2}$, 姚晓龙 ${ }^{1,2}$, 徐会显 ${ }^{1,2}, 弓^{2}$ 路 $^{1 * *}$ \\ (1: 中国科学院南京地理与湖泊研究所湖泊与环境国家重点实验室,南京 210008) \\ (2: 中国科学院大学, 北京 100049)
}

\begin{abstract}
摘 要: 反硝化 (Denitrification, DNF) 和硝酸盐异化还原为氨 (Dissimilatory Nitrate Reduction to Ammonium, DNRA) 是硝 酸盐异养还原的 2 个主要途径. 反硝化被认为是彻底去除水体氮负荷的主要过程; 而硝酸盐异化还原为氨则将水体中的 硝态氮转化为氨氮. 2 个过程均以硝酸盐为电子受体, 并存在相互竞争关系. 这 2 个过程的研究对理解湿地氮转化以及指 导湿地氮污染修复具有重要意义. 运用无扰动沉积物柱样流动培养、 ${ }^{15} \mathrm{NO}_{3}^{-}-\mathrm{N}$ 同位素示踪实验, 并采用氨氧化-膜接口质 谱仪联用 (OX/MIMS) 测定氨氮同位素产物的方法, 对鄱阳湖碟形湖湿地、巢湖重污染河流湿地、巢湖重污染湖泊湿地 3 种类型湿地沉积物一水界面的硝酸盐异养还原过程进行研究, 结果表明存在显著差异. 3 种类型湿地 DNF 速率的范围为 $(6.36 \pm 2.57) \sim(99.98 \pm 14.05) \mu \mathrm{mol} /\left(\mathrm{m}^{2} \cdot \mathrm{h}\right)$, DNRA 速率的范围为 $(0.51 \pm 0.45) \sim(79.82 \pm 6.08) \mu \mathrm{mol} /\left(\mathrm{m}^{2} \cdot \mathrm{h}\right)$. 在 3 种 类型湿地中, 随着氮污染程度加重, DNF 和 DNRA 速率均显著增加, 且 DNRA 过程在总的硝态氮异养还原中所占的比重 不断增大, 说明较高的硝酸盐负荷、较高的沉积物有机质含量更有利于 DNRA 过程的竞争. 而对反硝化方式的进一步研 究发现, 巢湖重污染河流、湖泊湿地主要以非耦合反硝化为主导过程, 而鄱阳湖碟形湖湿地则更倾向于以硝化过程堣合 控制的反硝化为主.
\end{abstract}

关键词: DNRA; 反硝化; 硝酸盐异养还原; 沉积物-水界面; 长江中下游; 巢湖; 鄱阳湖; 碟形湖湿地; 重污染河流湿地; 重 污染湖泊湿地

\section{Dissimilatory nitrate reduction processes between the sediment-water interface in three typical wetlands of middle and lower reaches of Yangtze River}

\author{
JIANG Xingyu ${ }^{1,2}$, YAO Xiaolong ${ }^{1,2}$, XU Huixian ${ }^{1,2} \&$ ZHANG Lu ${ }^{1 * *}$ \\ (1: State Key Laboratory of Lake Science and Environment Research, Nanjing Institute of Geography and Limnology, Chinese \\ Academy of Sciences, Nanjing 210008, P.R. China) \\ (2: University of Chinese Academy of Sciences, Beijing 100049, P.R.China)
}

\begin{abstract}
Denitrification(DNF) and dissimilation reduction to ammonium(DNRA) are two major pathways for the dissimilatory nitrate reduction processes. Denitrification is the dominant nitrogen loss process that removes nitrate in the water column, while DNRA reduces nitrate to ammonium which is still a biologically available form in aquatic environments. The nitrate served as electron acceptor during denitrification and DNRA processes, and the balance of two processes depends on environmental factors. The study on rates of denitrification and DNRA plays an important role in understanding the fate of nitrate and its dynamic. In this study, intact sediment cores were collected in three wetlands of Lake Poyang and Lake Chaohu. Denitrification and DNRA rates were determined by continuous-flow experiments combined with ${ }^{15} \mathrm{~N}$ isotope-tracing techniques to investigate their contributions to total nitrate reduction. The results showed that denitrification and DNRA rates ranged from $(6.36 \pm 2.57)$ to $(99.98 \pm 14.05) \mu \mathrm{mol} /\left(\mathrm{m}^{2} \cdot \mathrm{h}\right)$ and $(0.51 \pm 0.45)$ to $(79.82 \pm 6.08) \mu \mathrm{mol} /\left(\mathrm{m}^{2} \cdot \mathrm{h}\right)$, respectively. DNRA rates were enhanced significantly with increasing pollution degree as well as the DNRA/( DNF+DNRA) ratio, which indicates that the contribution of DNRA to total nitrate reduction in these wetlands was affected by nitrate loadings. Uncoupled nitrification-denitrification was the dominant nitrogen removal process in Lake Chaohu, and coupled denitrification was the dominant process in the Lake Poyang.
\end{abstract}

* 国家重点基础研究发展计划“973”项目 (2012CB417005) 和国家自然科学基金项目 (41271468)联合资助. 2015 11-16 收稿; 2016-04-28 收修改稿. 姜星宇(1991 ), 男,硕士研究生;E-mail : cui19910621@126.com.

** 通信作者; E-mail: luzhang@ niglas.ac.cn. 
Keywords: DNRA; denitrification; dissimilation reduction of nitrate; the sediment-water interface; middle and lower reaches of Yangtze River

湖泊、河流等生态系统中的氮污染已经成为全球范围内人们迫切关注的环境问题 ${ }^{[1-2]}$. 研究人员普遍认 为氮肥的大量施用以及固氮作物的大面积种植是导致河流、湖泊湿地氮负荷过高的主要原因 ${ }^{[3]}$. 水体中过 高的氮负荷使水质不断恶化,引发水体富营养化、缺氧面积不断扩大及蓝藻水华频繁暴发等一系列的环境 问题 ${ }^{[4-5]}$. 水体中氮的存在形态和转化过程对水环境质量和生态系统健康具有重要影响 ${ }^{[6]}$. 硝态氮是水体中 氮素的主要存在形态之一, 具有较强的迁移性和生物可利用性, 其迁移和转化一直是科研人员关注的重点. 湿地水体中硝态氮的去除途径主要有 2 种, 一是硝态氮被水中浮游植物或微生物吸收同化, 成为有机体的 组成部分; 二是在反硝化细菌的作用下经过呼吸反硝化过程转变为氮气迁移到大气中 ${ }^{[4-5,7]}$. 至今为止, 大多 数研究者仍旧认为生物同化作用和反硝化作用是湖泊、河流等湿地硝态氮最为主要的去除形式 ${ }^{[7]}$.

反硝化作用被认为是水体中至关重要的氮循环过程之一, 由于氮气不能被大多数生物所利用, 因而反 硝化作用是将氮素从水体中彻底去除的主要途径, 对降低水体氮负荷、修复氮污染具有十分重要的生态学 意义 ${ }^{[5]}$. 在反硝化过程中, 硝态氮作为在厌氧条件下有机物氧化过程最终的电子受体, 大部分的硝态氮被还 原成氮气, 还有很少的一部分以氧化亚氮的形式存在 ${ }^{[8]}$. 然而许多研究发现, 基于直接测定反硝化产物计算 得到的反硝化氮去除量经常不足实际硝态氮总去除量的一半 ${ }^{[9]}$. 许多研究将反硝化与硝态氮总去除量的差 异归因于 DNRA 过程 ${ }^{[7]}$. 作为另一种重要的硝态氮异氧还原过程, DNRA 不同于产生氮气 (或氧化亚氮) 的 反硝化过程, 它是在缺氧条件下将硝态氮异化还原为氨, 其产物氨氮是一种容易被生物利用的活性氮, 在湿 地系统中氨氮可以被植物、微生物吸收, 或经过硝化作用转化为硝态氮 ${ }^{[7]}$. 现今已知的 DNRA 途径有 2 种, 一是与微生物发酵有关; 二是与硫的氧化过程有关 ${ }^{[5]}$. 在还原条件下, DNRA 过程能够引发一个反馈回路, 使水体中的无机氮不断累积 ${ }^{[10]}$. DNRA 和反硝化过程有着共同的反应底物, 弄清它们在硝态氮异氧还原过 程中的相对重要性具有非常重要的环境意义, 它反映了有多少硝态氮会从水体中彻底移除, 而有多少硝态 氮转化为氨氮继续存在于水体氮循环过程当中 ${ }^{[11]}$. 因此探究 DNRA 和反硝化过程相对重要性的主要影响 因素, 探究随着氮负荷的增加硝态氮异氧还原各过程之间如何达到新的平衡, 有助于我们了解可被生物利 用的氮素在水体中的停留时间 ${ }^{[12]}$, 对理解湿地氮素转化、湿地水体营养水平以及氮污染控制等具有十分重 要的意义 ${ }^{[7]}$.

目前, 已有许多土壤生态系统中 DNRA 过程的研究 ${ }^{[13]}$, 对于湿地 DNRA 过程的有关研究还相对匮乏. 特别是相较于海洋及河口湿地, 人们对淡水湿地中的 DNRA 过程缺乏足够的认识, 而将 DNRA 与反硝化过 程联合研究则更为少见 ${ }^{[10]}$. DNRA 过程的研究中, 同位素示踪法是一种被普遍使用的有力方法, 简言之, 即 将 ${ }^{15} \mathrm{~N}$ 标记的硝酸盐作为示踪剂, 测定 DNRA 过程中产生的 ${ }^{15} \mathrm{NH}_{4}^{+}-\mathrm{N}$ 产物. 传统方法测定水体 ${ }^{15} \mathrm{NH}_{4}^{+}-\mathrm{N}$ 浓度 往往需要长时间的样品处理、复杂的操作过程以及大量的水样 ${ }^{[14]}$. 由于缺乏简便快速的方法来测定水体 中 ${ }^{15} \mathrm{NH}_{4}^{+}-\mathrm{N}$ 浓度, 较大地限制了对这一问题的研究 ${ }^{[12]}$. 本研究采用近年来出现的一种新方法, 即 OX/MIMS 法来快速测定 ${ }^{15} \mathrm{NH}_{4}^{+}-\mathrm{N}$ 浓度, 进而计算得到 DNRA 速率; 而反硝化过程的测定同样采用 ${ }^{15} \mathrm{~N}$ 标记的硝酸盐作 为示踪剂, 并采用同位素配对法 (IPT) 计算反硝化速率. 采用一种标记底物联合测定两种硝酸盐异养还原过 程,并比较它们在不同湿地类型沉积物一水界面对硝态氮去除和转化的各自贡献,也是本研究的一种尝试.

\section{1 材料与方法}

\section{1 样品采集和处理}

选择位于鄱阳湖 $\left(28^{\circ} 24^{\prime} \sim 29^{\circ} 46^{\prime} \mathrm{N}, 115^{\circ} 49^{\prime} \sim 116^{\circ} 46^{\prime} \mathrm{E}\right.$ ) 和巢湖 $\left(31^{\circ} 43^{\prime} \sim 32^{\circ} 25^{\prime} \mathrm{N}, 117^{\circ} 16^{\prime} \sim 117^{\circ} 51^{\prime} \mathrm{E}\right.$ ) 流域的湿地进行研究, 鄱阳湖和巢湖位于长江中下游地区均属于我国五大淡水湖泊. 其中, 鄱阳湖以大湖 池、中湖池等 9 个碟形湖湿地为代表, 属于鄱阳湖边缘性自然小湖泊, 冬、春季草滩湿地显露, 环境资源丰 富, 是鄱阳湖国家级鸟类自然保护区, 水质条件良好, 其地理、地貌和水文特征具有明显的典型性 ${ }^{[15]}$; 巢湖是 长江下游富营养化最为严重的湖泊之一, 尤其是西北部的人湖河流南汁河和十五里河受城市工业、生活污 水污染严重, 氮负荷高 ${ }^{[16]}$. 根据鄱阳湖、巢湖湿地不同的水质状况和污染程度, 将采样点湿地分为 3 种类型 
以研究其沉积物-水界面的硝态氮异养还原过程 (表 1). 样品采集分别于 2014 年 11 月和 2015 年 1 月完成. 在鄱阳湖 9 个碟形湖湿地中各选定 1 个代表性样点, 每个采样点采集 2 根柱样; 在巢湖分别于南淝河与十五 里河 2 条重污染河流以及重污染湖区各选定 1 个采样点, 每个采样点采集 3 根柱样 (泥柱深度 $15 \sim 20 \mathrm{~cm}$ ). 沉积物柱样均用有机玻璃柱状采样器( 高 $25 \mathrm{~cm}$ 、直径 $7.5 \mathrm{~cm}$ ) 采集, 两端用橡皮塞塞紧保存. 为保证采集的 沉积物有较好的同质性,所有柱样均在较小的区域内一次性采集,同时用 $25 \mathrm{~L}$ 聚乙烯桶采集采样点的上覆 水. 用多参数水质测定仪 (YSI 6600V2, 美国) 现场测定上覆水基本理化参数. 采集好的样品于当天运回实 验室分析和培养, 在运输过程中尽量避免沉积物界面的扰动. 反硝化和 DNRA 过程采用柱样沉积物流动培 养法进行研究.

表 1 采样点水体基本理化参数

Tab.1 General characteristics of the water column in three typical wetlands

\begin{tabular}{lllcccc}
\hline 3 种类型湿地 & 采样点 & 经纬度 & 水温/ ${ }^{\circ} \mathrm{C}$ & $\mathrm{DO} /(\mathrm{mg} / \mathrm{L})$ & $\mathrm{pH}$ 值 & $\mathrm{ORP} / \mathrm{mV}$ \\
\hline 鄱阳湖碟形湖湿地 & 大湖池 & $29^{\circ} 9^{\prime} 28^{\prime \prime} \mathrm{N}, 115^{\circ} 56^{\prime} 42^{\prime \prime} \mathrm{E}$ & 7.34 & 8.66 & 5.78 & 172 \\
& 象湖 & $29^{\circ} 11^{\prime} 15^{\prime \prime} \mathrm{N}, 116^{\circ} 0^{\prime} 46^{\prime \prime} \mathrm{E}$ & 6.60 & 8.79 & 6.69 & 85 \\
& 中湖池 & $29^{\circ} 9^{\prime} 16^{\prime \prime} \mathrm{N}, 116^{\circ} 0^{\prime} 26^{\prime \prime} \mathrm{E}$ & 9.19 & 8.61 & 7.49 & 169 \\
& 蛙湖 & $29^{\circ} 11^{\prime} 53^{\prime \prime} \mathrm{N}, 115^{\circ} 58^{\prime} 35^{\prime \prime} \mathrm{E}$ & 8.15 & 10.03 & 7.99 & 127 \\
& 朱市湖 & $29^{\circ} 10^{\prime} 47^{\prime \prime} \mathrm{N}, 115^{\circ} 58^{\prime} 59^{\prime \prime} \mathrm{E}$ & 9.51 & 7.93 & 8.33 & 126 \\
& 梅西湖 & $29^{\circ} 12^{\prime} 52^{\prime \prime} \mathrm{N}, 115^{\circ} 3^{\prime} 38^{\prime \prime} \mathrm{E}$ & 7.55 & 9.65 & 5.51 & 223 \\
& 大汉湖 & $29^{\circ} 10^{\prime} 34^{\prime \prime} \mathrm{N}, 116^{\circ} 2^{\prime} 45^{\prime \prime} \mathrm{E}$ & 11.86 & 10.17 & 6.04 & 238 \\
& 常湖池 & $29^{\circ} 8^{\prime} 8^{\prime \prime} \mathrm{N}, 115^{\circ} 59^{\prime} 2^{\prime \prime} \mathrm{E}$ & 6.95 & 11.29 & 6.42 & 172 \\
& 沙湖 & $29^{\circ} 10^{\prime} 40^{\prime \prime} \mathrm{N}, 115^{\circ} 55^{\prime} 6^{\prime \prime} \mathrm{E}$ & 10.52 & 7.88 & 6.72 & 216 \\
巢湖重污染河流湿地 & 十五里河 & $31^{\circ} 43^{\prime} 58^{\prime \prime} \mathrm{N}, 117^{\circ} 19^{\prime} 47^{\prime \prime} \mathrm{E}$ & 9.12 & 1.93 & 6.80 & 102.9 \\
& 南淝河 & $31^{\circ} 45^{\prime} 47^{\prime \prime} \mathrm{N}, 117^{\circ} 25^{\prime} 55^{\prime \prime} \mathrm{E}$ & 7.58 & 3.77 & 7.02 & 131.5 \\
巢湖重污染湖泊湿地 & 巢湖 & $31^{\circ} 40^{\prime} 22^{\prime \prime} \mathrm{N}, 117^{\circ} 22^{\prime} 46^{\prime \prime} \mathrm{E}$ & 5.18 & 10.53 & 7.92 & 112.9 \\
\hline
\end{tabular}

\section{2 室内培养实验}

样柱运回实验室后垂直放置, 打开上端橡皮塞, 用注射器沿管壁尽量无扰动地注满采集来的现场水样, 恒温 $\left(10^{\circ} \mathrm{C}\right)$ 水浴过夜, 以去除运输过程中因短暂密封造成的水体溶解氧下降等因素的影响. 为避免沉积物 和水体中藻类光合作用对反硝化和 DNRA 过程的影响, 样柱在培养过程中用铝箔包裹. 对上述样柱进行无 顶空流动培养. 培养系统包括带有密封圈的活塞、进出水管以及提供恒定进水流速的蠕动泵. 进水管距沉积 物-水界面约 $1 \mathrm{~cm}$, 进水管低于出水管, 通过蠕动洜将水箱中于采样点采集的水样以恒定的流速 $(1 \mathrm{ml} / \mathrm{min})$ 㫳人柱样中, 使流动培养的进出水循环形成垂直及水平方向的混合水流, 使培养体系中上覆水充分混合, 并 通过持续流动保持上覆水中离子浓度的稳定. 经过 $24 \mathrm{~h}$ 的预培养后, 采集培养体系的进出水样, 经醋酸纤维 滤膜 ( 孔径 $0.2 \mu \mathrm{m}$ ) 过滤后, 进行溶解性无机氮的分析, 用以计算样柱沉积物一水界面无机氮的通量. 样品采 集完成后向进水水箱中加人 ${ }^{15} \mathrm{NO}_{3}^{-}-\mathrm{N}$ 同位素 (Cambridge Isotope Laboratories, Inc) 溶液, 使水箱中 ${ }^{15} \mathrm{NO}_{3}^{-}-\mathrm{N}$ 浓 度约为 $100 \mu \mathrm{mol} / \mathrm{L}$, 并继续经 $24 \mathrm{~h}$ 培养后, 再次采集样品. 其中, 进水水样用注射器小心采集, 在无气泡产生 的情况下装满并充分溢流至细长的磨口玻璃采集管中, 出水水样直接用磨口玻璃管溢流收集, 均加人 $200 \mu \mathrm{l}$ $50 \% \mathrm{ZnCl}_{2}$ 以对微生物进行灭活, 在 $4{ }^{\circ} \mathrm{C}$ 冰箱保存用以分析水样中由反硝化过程产生的溶解性气体 ${ }^{28} \mathrm{~N}_{2}$ 、 ${ }^{29} \mathrm{~N}_{2} 、{ }^{30} \mathrm{~N}_{2}$ ) 含量. 与此同时, 用同样的方法收集进、出水样, 将得到的水样过滤后通人氦气, 吹扫 $20 \mathrm{~min}$, 以去 除水样中溶解的同位素气体 $\left({ }^{29} \mathrm{~N}_{2} 、{ }^{30} \mathrm{~N}_{2}\right)$. 把得到的样品转移到 $12 \mathrm{ml}$ 的 labco 气密瓶中, 确保瓶中无气体残 留后用带橡胶垫的瓶盖密封, 以防止样品被大气污染, 随后立即加人 $0.2 \mathrm{ml}$ 的氧化剂 (次溴酸盐的碘溶液), 在 $4{ }^{\circ} \mathrm{C}$ 冰箱保存用以分析由 DNRA 过程产生的铵态氮 $\left(\mathrm{NH}_{4}^{+}-\mathrm{N}\right)$ 浓度.

本研究用膜接口质谱仪 (MIMS) 测定水样中由反硝化过程产生的溶解性气体含量, 根据测定结果通过 同位素配对法计算实际和潜在反硝化速率. 采用氨氧化技术与膜接口质谱分析相结合的方法 (OX/MIMS) 来测定 ${ }^{15} \mathrm{NH}_{4}^{+}-\mathrm{N}$ 浓度, 进而计算 DNRA 速率. 氧化剂的制备 ${ }^{[17]}$ : 将液溴逐滴加人到氢氧化钠溶液中, 不断摚 拌并使得整个反应在低于 $5^{\circ} \mathrm{C}$ 的条件下进行; 将得到的溶液冷冻保存以便使生成的溴化钠结晶吸出, 用过滤 
得到的上清液与同体积的碘化钾溶液混合以便稳定保存, 方便使用. 用氧化剂将水样中的 $\mathrm{NH}_{4}^{+}-\mathrm{N}$ 氧化为 $\mathrm{N}_{2}$, 再用膜接口质谱仪 (MIMS) 测定水样中可溶性气体 $\left({ }^{28} \mathrm{~N}_{2} 、{ }^{29} \mathrm{~N}_{2} 、{ }^{30} \mathrm{~N}_{2}\right)$ 含量, 根据 ${ }^{29} \mathrm{~N}_{2} 、{ }^{30} \mathrm{~N}_{2}$ 含量计算出由 DNRA 过程产生的 ${ }^{15} \mathrm{NH}_{4}^{+}-\mathrm{N}$ 浓度, 进而得到实际和潜在 DNRA 速率. 使用连续流动分析仪 ( SKALAR $\mathrm{SAN}^{++}$) 测定水样中溶解性无机氮 (硝态氮 $\left(\mathrm{NO}_{3}^{-}-\mathrm{N}\right)$ 、亚硝态氮 $\left(\mathrm{NO}_{2}^{-}-\mathrm{N}\right)$ 和 $\mathrm{NH}_{4}^{+}-\mathrm{N}$ 浓度).

\section{3 营养盐通量和反硝化、DNRA 速率的计算方法}

在流动培养实验中, $\mathrm{NH}_{4}^{+}-\mathrm{N} 、 \mathrm{NO}_{3}^{-}-\mathrm{N}$ 在沉积物-水界面的通量 $\left(F_{\mathrm{a}} 、 F_{\mathrm{n}}\right)$ 计算公式为 ${ }^{[18]}$ :

$$
F=\left(C-C_{0}\right) \cdot v / S
$$

式中, $F$ 为 $\mathrm{NH}_{4}^{+}-\mathrm{N} 、 \mathrm{NO}_{3}^{-}-\mathrm{N}$ 的通量 $\left(\mu \mathrm{mol} /\left(\mathrm{m}^{2} \cdot \mathrm{h}\right)\right), C_{0} 、 C$ 为取样时进、出水中营养盐浓度 $(\mu \mathrm{mol} / \mathrm{L}), v$ 为蠕 动百流速 $(\mathrm{ml} / \mathrm{min}), S$ 为柱样中沉积物 - 水界面面积 $\left(\mathrm{m}^{2}\right)$.

使用公式 (1) 可以求出 $\mathrm{N}_{2}$ 在沉积物一水界面的释放速率 $\left(r_{29} 、 r_{30}\right): r_{n}=\left(c-c_{0}\right) \cdot v / S$, 此时式中 $r_{n}$ 为氮 气 $\left({ }^{29} \mathrm{~N}_{2} 、{ }^{30} \mathrm{~N}_{2}\right)$ 释放速率 $\left(\mu \mathrm{mol} /\left(\mathrm{m}^{2} \cdot \mathrm{h}\right)\right) ; c_{0} 、 c$ 分别为取样时进出水中氮气含量 $(\mu \mathrm{mol} / \mathrm{L})$; 其余各项与式 (1) 相同. 通过 ${ }^{29} \mathrm{~N}_{2}\left(r_{29}\right)$ 和 ${ }^{30} \mathrm{~N}_{2}\left(r_{30}\right)$ 的产生速率可以算出 ${ }^{15} \mathrm{NO}_{3}^{-}-\mathrm{N}$ 的反硝化速率 $\left(D_{15}\right)$ :

$$
D_{15}=r_{29}+2 r_{30}
$$

根据同位素配对法 ${ }^{[19]}$ 可以求算出 ${ }^{14} \mathrm{NO}_{3}^{-}-\mathrm{N}$ 的反硝化速率 $\left(D_{14}\right)$ :

$$
D_{14}=D_{15} \cdot\left(r_{29} / 2 r_{30}\right)
$$

而耦合反硝化速率 $\left(D_{\mathrm{n}}\right)$ 和非耦合反硝化速率 $\left(D_{\mathrm{w}}\right)$ 公式为 ${ }^{[20]}$ :

$$
\begin{gathered}
D_{\mathrm{pot}}=D_{14}+D_{15} \\
D_{\mathrm{w}}^{\text {tot }}=D_{15} / \varepsilon \\
\varepsilon=\left(\left[\mathrm{NO}_{3}^{-}\right]_{\mathrm{a}}-\left[\mathrm{NO}_{3}^{-}\right]_{\mathrm{b}}\right) /\left[\mathrm{NO}_{3}^{-}\right]_{\mathrm{a}} \\
D_{\mathrm{n}}=D_{\mathrm{pot}}-D_{\mathrm{w}}^{\text {tot }} \\
D_{\mathrm{w}}=D_{\mathrm{w}}^{\text {tot }} \cdot(1-\varepsilon)
\end{gathered}
$$

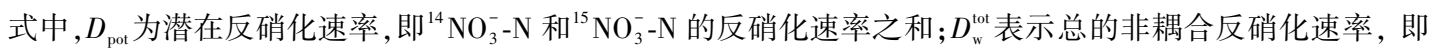
上覆水中的反硝化速率; $\varepsilon$ 代表培养实验中 ${ }^{15} \mathrm{NO}_{3}^{-}-\mathrm{N}$ 的丰度 (无量纲), 下标 $\mathrm{a}$ 和 $\mathrm{b}$ 表示添加同位素之后和 之前.

DNRA 速率公式为 ${ }^{[21]}$ :

$$
\begin{gathered}
{\left[{ }^{15} \mathrm{NH}_{4}^{+}\right]=r_{\mathrm{DNRA}}^{29}+2 r_{\mathrm{DNRA}}^{30}} \\
R_{\mathrm{DNRA}}^{15}=\left(\left[{ }^{15} \mathrm{NH}_{4}^{+}\right]_{\text {outflow }}-\left[{ }^{15} \mathrm{NH}_{4}^{+}\right]_{\text {inflow }}\right) \cdot v / S
\end{gathered}
$$

式中, $\left[{ }^{15} \mathrm{NH}_{4}^{+}\right]$为水体中的 ${ }^{15} \mathrm{NH}_{4}^{+}-\mathrm{N}$ 浓度, $r_{\mathrm{DNRA}}^{n}$ 表示由 $\mathrm{NH}_{4}^{+}-\mathrm{N}$ 氧化所产生的 ${ }^{29} \mathrm{~N}_{2} 、{ }^{30} \mathrm{~N}_{2}$ 浓度, 其中 2 个 ${ }^{15} \mathrm{NH}_{4}^{+}$被 同时氧化产生 ${ }^{30} \mathrm{~N}_{2},{ }^{15} \mathrm{NH}_{4}^{+}$和 ${ }^{14} \mathrm{NH}_{4}^{+}$同时氧化产生 ${ }^{29} \mathrm{~N}_{2} . R_{\text {DNRA }}^{15}{ }^{15} \mathrm{NO}_{3}^{-}-\mathrm{N}$ 的 DNRA 速率. 假设 DNRA 过程和反 硝化过程发生在相同的沉积物环境中,则实际的 DNRA 速率计算公式为 ${ }^{[22]}$ :

$$
R_{\mathrm{DNRA}}^{14}=R_{\mathrm{DNRA}}^{15} \cdot\left(D_{14} / D_{15}\right)
$$

\section{2 结果与分析}

\section{1 上覆水和沉积物的理化特性}

在 3 种类型湿地的上覆水中, $\mathrm{NO}_{3}^{-}-\mathrm{N}$ 浓度范围为 $6.15 \sim 479.76 \mu \mathrm{mol} / \mathrm{L}$, 巢湖重污染河流湿地和重污染湖 泊湿地上覆水中 $\mathrm{NO}_{3}^{-}-\mathrm{N}$ 浓度 $(374.80 \pm 104.96$ 和 $238.95 \pm 38.27 \mu \mathrm{mol} / \mathrm{L}$, 均值 \pm 标准偏差, 下同) 显著高于鄱阳 湖碟形湖湿地 $(19.94 \pm 17.68 \mu \mathrm{mol} / \mathrm{L})(P<0.05)$; 重污染河流湿地上覆水中 $\mathrm{NH}_{4}^{+}-\mathrm{N}$ 浓度最高 $(749.05 \pm 83.68$ $\mu \mathrm{mol} / \mathrm{L})$, 相比于重污染湖泊湿地和碟形湖湿地要高出 1 个数量级; $\mathrm{NO}_{2}^{-}-\mathrm{N}$ 浓度与 $\mathrm{NO}_{3}^{-}-\mathrm{N} 、 \mathrm{NH}_{4}^{+}-\mathrm{N}$ 浓度变化 一致, 均表现为重污染河流湿地 $>$ 重污染湖泊湿地>碟形湖湿地 $(P<0.05)$; 沉积物中有机质含量用烧失量表 示, 巢湖重污染河流、湖泊湿地的有机质含量要大于碟形湖湿地 (表 2 ). 
表 2 上覆水和沉积物的理化性质

Tab.2 Physicochemical parameters of the overlying water and the sediment

\begin{tabular}{lccccc}
\hline & 理化因子 & & 碟形湖湿地 & 重污染河流湿地 & 重污染湖泊湿地 \\
\hline 上覆水 & $\mathrm{NO}_{3}^{-}-\mathrm{N} /$ & 范围 & $6.15 \sim 66.03$ & $268.55 \sim 479.76$ & $228.95 \sim 311.44$ \\
& $(\mu \mathrm{mol} / \mathrm{L})$ & 均值士标准差 & $19.94 \pm 17.68$ & $374.80 \pm 104.96$ & $238.95 \pm 38.27$ \\
& $\mathrm{NO}_{2}^{-}-\mathrm{N} /$ & 范围 & $0.24 \sim 3.94$ & $38.98 \sim 53.04$ & $0.20 \sim 2.81$ \\
& $(\mu \mathrm{mol} / \mathrm{L})$ & 均值士标准差 & $0.94 \pm 1.13$ & $44.95 \pm 5.93$ & $1.50 \pm 1.30$ \\
& $\mathrm{NH}_{4}^{+}-\mathrm{N} /$ & 范围 & $4.36 \sim 57.29$ & $630.71 \sim 809.29$ & $84.57 \sim 92.85$ \\
表层沉积物 & $(\mu \mathrm{mol} / \mathrm{L})$ & 均值士标准差 & $31.71 \pm 17.40$ & $749.05 \pm 83.68$ & $88.71 \pm 4.14$ \\
& 有机质含量 ${ }^{1)} /$ & 范围 & $1.83 \sim 7.24$ & $6.03 \sim 6.84$ & $5.41 \sim 8.73$ \\
& $\%$ & 均值士标准差 & $4.10 \pm 1.62$ & $6.46 \pm 0.33$ & $7.07 \pm 1.66$ \\
\hline
\end{tabular}

1) 巢湖重污染河流湿地、湖泊湿地的有机质含量数据来源于文献 [23].

\section{2 沉积物一水界面营养盐通量}

沉积物-水界面 $\mathrm{NH}_{4}^{+}-\mathrm{N}$ 净通量范围为-300.24 286.18 $\mu \mathrm{mol} /\left(\mathrm{m}^{2} \cdot \mathrm{h}\right)$. 重污染河流湿地和湖泊湿地沉 积物均表现为 $\mathrm{NH}_{4}^{+}-\mathrm{N}$ 的源, 即 $\mathrm{NH}_{4}^{+}-\mathrm{N}$ 从沉积物释放到上覆水中, 其中重污染河流湿地沉积物 $\mathrm{NH}_{4}^{+}-\mathrm{N}$ 通量 $\left(240.82 \pm 88.20 \mu \mathrm{mol} /\left(\mathrm{m}^{2} \cdot \mathrm{h}\right)\right)$ 高于重污染湖泊湿地 $\left(107.19 \pm 55.78 \mu \mathrm{mol} /\left(\mathrm{m}^{2} \cdot \mathrm{h}\right)\right)$; 鄱阳湖碟形湖湿地沉 积物 $\mathrm{NH}_{4}^{+}-\mathrm{N}$ 通量 $\left(-106.12 \pm 134.69 \mu \mathrm{mol} /\left(\mathrm{m}^{2} \cdot \mathrm{h}\right)\right)$ 具有较大的空间差异性,但大部分表现为 $\mathrm{NH}_{4}^{+}-\mathrm{N}$ 的汇. $\mathrm{NO}_{3}^{-}-\mathrm{N}$ 通量同样具有较大的空间差异, 其范围为 $-337.49 \sim 110.74 \mu \mathrm{mol} /\left(\mathrm{m}^{2} \cdot \mathrm{h}\right)$, 但空间差异规律与 $\mathrm{NH}_{4}^{+}-\mathrm{N}$ 相反, 重污染湿地沉积物表现为 $\mathrm{NO}_{3}^{-}-\mathrm{N}$ 的汇 ( 重污染河流湿地沉积物为 $-277.27 \pm 88.92 \mu \mathrm{mol} /\left(\mathrm{m}^{2} \cdot \mathrm{h}\right.$ ), 重污 染湖泊湿地沉积物为 $\left.-337.49 \pm 73.44 \mu \mathrm{mol} /\left(\mathrm{m}^{2} \cdot \mathrm{h}\right)\right)$; 而碟形湖湿地 $\left(48.82 \pm 63.55 \mu \mathrm{mol} /\left(\mathrm{m}^{2} \cdot \mathrm{h}\right)\right)$ 总体上 则表现为 $\mathrm{NO}_{3}^{-}-\mathrm{N}$ 的源. 总溶解性无机氮 (DIN) 通量为 $\mathrm{NH}_{4}^{+}-\mathrm{N} 、 \mathrm{NO}_{2}^{-}-\mathrm{N}$ 和 $\mathrm{NO}_{3}^{-}-\mathrm{N}$ 三者净通量之和,范围为 $-229.91 \sim 195.47 \mu \mathrm{mol} /\left(\mathrm{m}^{2} \cdot \mathrm{h}\right)$, 3 种类型湿地沉积物均表现为 DIN 的汇, 其中, 重污染河流湿地的 DIN 通 量 $\left(-221.42 \pm 150.02 \mu \mathrm{mol} /\left(\mathrm{m}^{2} \cdot \mathrm{h}\right)\right)$ 高于重污染湖泊湿地 $\left(-49.30 \pm 20.13 \mu \mathrm{mol} /\left(\mathrm{m}^{2} \cdot \mathrm{h}\right)\right)$ 和碟形湖湿地 $\left(-39.75 \pm 127.26 \mu \mathrm{mol} /\left(\mathrm{m}^{2} \cdot \mathrm{h}\right)\right)$ (图 1).

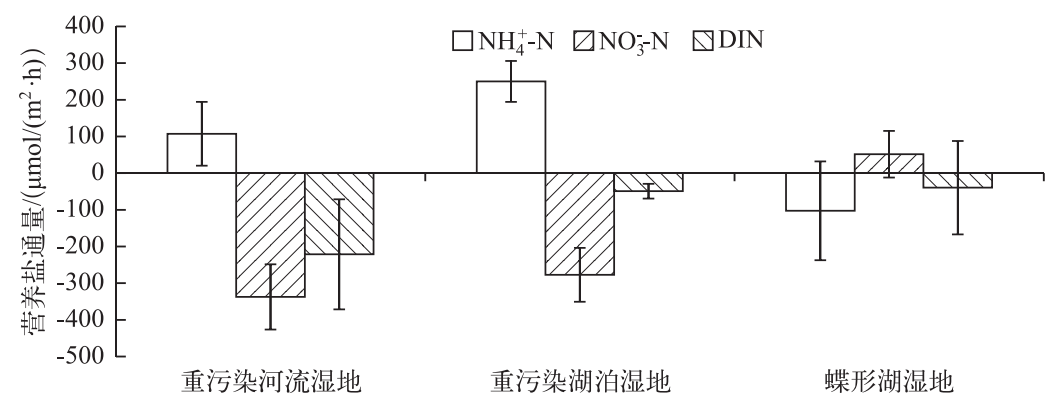

图 13 种类型湿地沉积物-水界面营养盐通量

Fig. 1 Nutrient fluxes between the sediment-water interface in three typical wetlands

\section{3 反硝化速率,耦合反硝化、非耦合反硝化速率以及 DNRA 速率}

根据反硝化方式的不同可以将反硝化分为耦合反硝化和非耦合反硝化. 耦合反硝化即耦合硝化一反硝 化脱氮过程, 反应所需的硝态氮主要是由沉积物中的硝化作用产生, 而非耦合反硝化所需的硝态氮主要来 自于上覆水 ${ }^{[2]}$. 巢湖重污染湿地沉积物的反硝化速率远高于鄱阳湖碟形湖湿地, 其中重污染河流湿地沉积 物的反硝化速率 $\left(99.98 \pm 14.05 \mu \mathrm{mol} /\left(\mathrm{m}^{2} \cdot \mathrm{h}\right)\right)$ 略高于重污染湖泊湿地 $\left(63.67 \pm 12.84 \mu \mathrm{mol} /\left(\mathrm{m}^{2} \cdot \mathrm{h}\right)\right)$; 而碟 形湖湿地沉积物的反硝化速率只有 $6.36 \pm 2.57 \mu \mathrm{mol} /\left(\mathrm{m}^{2} \cdot \mathrm{h}\right)(P<0.01)$. 巢湖重污染湿地沉积物的 $D_{\mathrm{n}}$ 和 $D_{\mathrm{w}}$ 均高于鄱阳湖碟形湖湿地 $(P<0.01 ; P<0.01)$, 但它们在反硝化速率中所占的比例却有很大差别. 在重污染 河流湿地和重污染湖泊湿地反硝化过程中 $D_{\mathrm{w}}$ 占据主导地位, 分别占反硝化速率的 $74 \%$ 和 $83 \%$, 其中 $D_{\mathrm{n}}$ 分别 
为 $25.74 \pm 6.95$ 和 $10.85 \pm 1.93 \mu \mathrm{mol} /\left(\mathrm{m}^{2} \cdot \mathrm{h}\right) ; D_{\mathrm{w}}$ 分别为 $74.24 \pm 11.13$ 和 $52.82 \pm 10.95 \mu \mathrm{mol} /\left(\mathrm{m}^{2} \cdot \mathrm{h}\right)$. 而在碟 形湖湿地沉积物中 $D_{\mathrm{n}}$ 对反硝化的贡献更大, 占总反硝化速率的 $61 \%\left(D_{\mathrm{n}}\right.$ 为 $3.99 \pm 2.16 \mu \mathrm{mol} /\left(\mathrm{m}^{2} \cdot \mathrm{h}\right), D_{\mathrm{w}}$ 为 $2.64 \pm 2.06 \mu \mathrm{mol} /\left(\mathrm{m}^{2} \cdot \mathrm{h}\right)$ ) (图 2a).
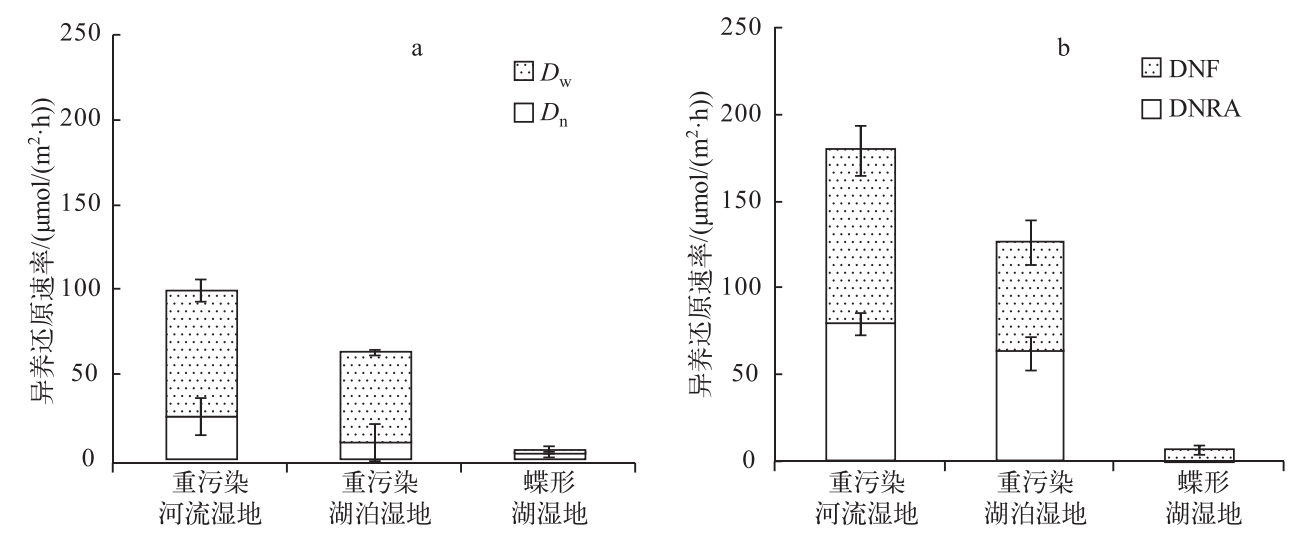

图 $2 D_{\mathrm{n}} 、 D_{\mathrm{w}}(\mathrm{a})$ 以及 $\mathrm{DNF} 、 \operatorname{DNRA}(\mathrm{b})$ 速率

Fig. $2 D_{\mathrm{n}}, D_{\mathrm{w}}(\mathrm{a})$ and the rates of $\mathrm{DNF}, \operatorname{DNRA}(\mathrm{b})$

在 3 种类型的湿地沉积物中, 重污染河流湿地总的硝酸盐异养还原速率 (DNF+DNRA) 要高于重污染湖 泊湿地, 且远高于碟形湖湿地 (图 2b). 与 DNF 速率相比, 不同类型湿地沉积物的 DNRA 速率差异更为明显. 重污染河流湿地和重污染湖泊湿地沉积物 DNRA 速率高出碟形湖湿地 2 个数量级. 其中重污染河流湿地的 DNRA 速率 $\left(79.82 \pm 6.08 \mu \mathrm{mol} /\left(\mathrm{m}^{2} \cdot \mathrm{h}\right)\right)$ 要高于重污染湖泊湿地 $\left(63.05 \pm 9.80 \mu \mathrm{mol} /\left(\mathrm{m}^{2} \cdot \mathrm{h}\right)\right)$, 而碟形湖湿 地沉积物的 DNRA 速率仅为 $0.51 \pm 0.45 \mu \mathrm{mol} /\left(\mathrm{m}^{2} \cdot \mathrm{h}\right)(P<0.01)$.

\section{3 讨论}

\section{1 沉积物-水界面营养盐通量}

重污染河流湿地和重污染湖泊湿地沉积物一水界面具有较高的氨氮释放通量. 导致该类型湿地水体中 氨氮不断累积的原因可能有以下几个方面: 在重污染湿地厌氧环境下微生物的同化效率低, 且厌氧矿化可 能会比好氧矿化作用产生更多的氨氮 ${ }^{[9,24]}$; 重污染河流湿地和重污染湖泊湿地 $D_{\mathrm{n}}$ 在总反硝化中所占比例只 有 $26 \%$ 和 $17 \%$, 而在碟形湖湿地中则为 $59 \%$, 从侧面反映出重污染湿地的硝化作用受到抑制; 此外, 在重污 染湿地沉积物一水界面中 DNRA 过程对氨的再生也有一定的贡献作用, 重污染河流、湖泊湿地 DNRA 速率远 大于碟形湖湿地,使重污染湿地沉积物总体表现为 $\mathrm{NH}_{4}^{+}-\mathrm{N}$ 的源.

在 3 种类型的湿地中, $\mathrm{NO}_{3}^{-}-\mathrm{N}$ 通量与 $\mathrm{NH}_{4}^{+}-\mathrm{N}$ 通量的空间差异正好相反. 在重污染河流、湖泊湿地中, 沉 积物-水界面的 $\mathrm{NO}_{3}^{-}-\mathrm{N}$ 通量为负值, 与碟形湖湿地相比, 较大的反硝化以及 DNRA 速率使得硝态氮不断从 水体中移除, 在重污染河流湿地和重污染湖泊湿地中, $\mathrm{NO}_{3}^{-}-\mathrm{N}$ 总的异养还原速率 $(\mathrm{DNF}+\mathrm{DNRA})$ 分别占 $\mathrm{NO}_{3}^{-}-\mathrm{N}$ 净通量的 $53 \%$ 和 $46 \%, \mathrm{NO}_{3}^{-}-\mathrm{N}$ 的异养还原过程是重污染湿地硝酸盐去除的重要原因. 在碟形湖湿地 中, 相对较低的 DNF 以及 DNRA 速率以及较为有利的硝化反应条件使得该类型湿地沉积物总体表现为 $\mathrm{NO}_{3}^{-}-\mathrm{N}$ 的源.

在重污染河流湿地、重污染湖泊湿地和碟形湖湿地中溶解态无机氮通量均为负值, 总体上均表现为溶 解态无机氮不断从上覆水迁移到沉积物中. 氮循环各个过程相对重要性的不同造成了不同类型无机氮的累 积或移除. 沉积物是无机氮的源或汇受多种因素的影响, $\mathrm{NO}_{3}^{-}-\mathrm{N}$ 和 $\mathrm{NH}_{4}^{+}-\mathrm{N}$ 通量的差异主要受到矿化作用、硝 化作用、反硝化作用、DNRA 作用, 底栖生物的吸收与排泄以及沉积物氮库的富集交换等因素的影响 ${ }^{[25-27]}$.

\section{2 耦合反硝化与非耦合反硝化}

3 种不同类型湿地沉积物的反硝化方式, 即耦合反硝化与非耦合反硝化的相对重要性同样存在着明显 
的差别. 在 3 种类型湿地的所有采样点中, $D_{\mathrm{w}}$ 与沉积物上覆水中 $\mathrm{NO}_{3}^{-}-\mathrm{N}$ 浓度之间具有明显的线性关系 $(r=$ $0.98, P<0.001$ ) (图 3a). 在碟形湖湿地中, 随着上覆水 $\mathrm{NO}_{3}^{-}-\mathrm{N}$ 浓度的不断增加, 除了 $D_{\mathrm{w}}$ 呈线性递增外, $D_{\mathrm{w}}$ 在 反硝化中所占的比例 $\left(D_{\mathrm{w}} / D_{14}\right)$ 也不断增大 $(r=0.84, P<0.001)$ ( 图 $3 \mathrm{~b}$ ), 上覆水中 $\mathrm{NO}_{3}^{-}-\mathrm{N}$ 浓度是控制反硝化 方式的决定性因素; 而在巢湖重污染湿地中并没有得到类似结果. 在重污染湿地沉积物中硝化作用受到抑 制进而影响 $D_{\mathrm{n}}$, 这可能是导致 $D_{\mathrm{w}} / D_{14}$ 与上覆水 $\mathrm{NO}_{3}^{-}-\mathrm{N}$ 浓度之间不存在明显线性关系的主要原因.
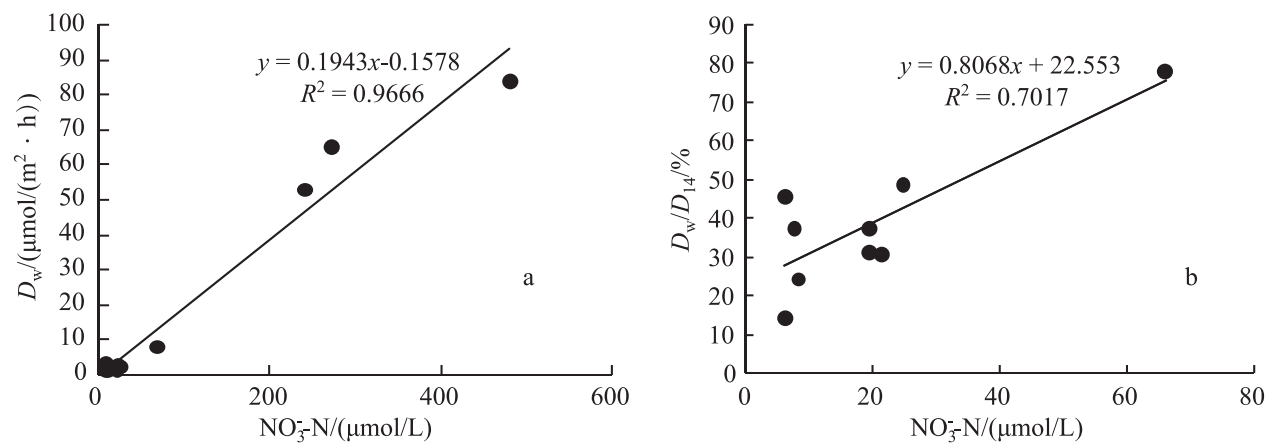

图 3 所有类型湿地 $D_{\mathrm{w}}(\mathrm{a})$ 、碟形湖湿地 $D_{\mathrm{w}} / D_{14}(\mathrm{~b})$ 与上覆水 $\mathrm{NO}_{3}^{-}-\mathrm{N}$ 浓度之间的关系

Fig. 3 The relationship between the $D_{w}$ and $\mathrm{NO}_{3}^{-}-\mathrm{N}$ concentration in overlying water in all types of wetland(a), the relationship between the $D_{\mathrm{w}} / D_{14}$ and

$\mathrm{NO}_{3}^{-}-\mathrm{N}$ concentration in overlying water in the saucer lake wetland of Lake Poyang ( b )

\section{3 不同类型湿地的反硝化和 DNRA 过程}

与国际上已有的一些对湖泊、河流等湿 地反硝化和 DNRA 过程的研究 ${ }^{[10-11,22,28]}$ 相比, 本研究中重污染河流湿地和重污染湖泊湿地 的反硝化速率在文献值范围之内, 但相对较 低. 推测其原因: 实验是在冬季进行,较低的 温度抑制了反硝化细菌的活性 ${ }^{[21]}$; 而碟形湖 湿地反硝化速率与其它类似的结果相比约低 1 个数量级, 除了可能的温度因素外, 反应底 物 $\left(\mathrm{NO}_{3}^{-}-\mathrm{N}\right)$ 的相对匮乏可能是另一重要原因. 硝态氮浓度是限制反硝化速率的主要因 素 ${ }^{[29]}$,通过对在 3 种类型湿地采集的样品进 行实验分析, 反硝化速率与上覆水中 $\mathrm{NO}_{3}^{-}-\mathrm{N}$ 浓度呈明显的线性关系 (图 4). 在不同类型

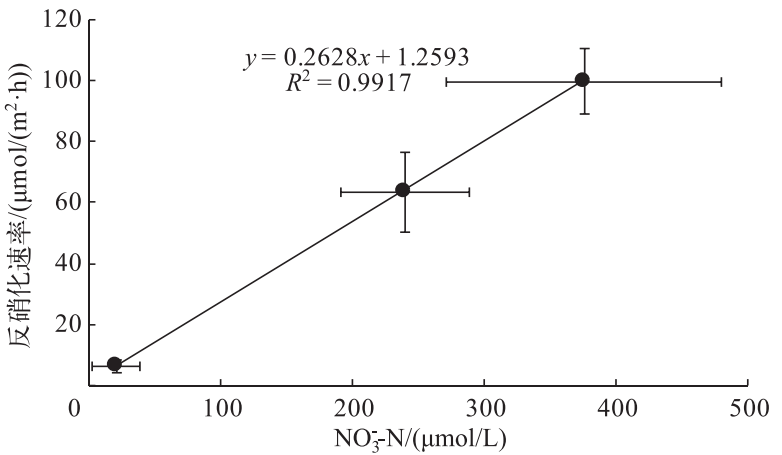

图 4 沉积物反硝化速率与上覆水 $\mathrm{NO}_{3}^{-}-\mathrm{N}$ 浓度的关系

Fig.4 The relationship between denitrification rates and $\mathrm{NO}_{3}^{-}-\mathrm{N}$ concentration in the overlying water 湿地中, 反硝化是最为主要的硝酸盐异氧还原过程, 反硝化速率约占总 $\mathrm{NO}_{3}^{-}-\mathrm{N}$ 异养还原速率的 $50 \%$ \% $93 \%$.

巢湖重污染河流湿地和重污染湖泊湿地 DNRA 速率与文献值相比相对较高, 更值得注意的是, DNRA 速率在总的硝酸盐异养还原过程中所占比例也较高. 而碟形湖湿地 DNRA 速率与绝大多数的研究相似 (表 3). 在巢湖重污染湿地沉积物中, 较高的有机质含量有利于硫化氢等还原态硫化物的大量生成, 而硫化物在 $\mathrm{NO}_{3}^{-}-\mathrm{N}$ 的异养还原过程中扮演了重要的角色 ${ }^{[21]}$ : 硫化物能够抑制反硝化酶的活性; DNRA 过程与硫的氧化 过程密切相关 ${ }^{[30]}$, 硝酸盐可作为电子受体将还原态的硫氧化为硫酸盐; 而硫酸盐可与硝酸盐竞争作为电子 受体,会进一步抑制反硝化作用,从而有利于 DNRA 过程与 DNF 过程的竞争. Burgin 等 ${ }^{[30]}$ 对美国密歇根洲 的 9 个湿地、9 条河流和 3 个小型湖泊的研究中发现, DNRA 与硫氧化具有密切相关性, DNRA 在 $\mathrm{NO}_{3}^{-}-\mathrm{N}$ 异 养还原过程中所占比重与本文结果类似. 从能量利用效率的角度看待这一问题, Tiedje ${ }^{[31]}$ 认为, 在可利用碳 源丰富的环境中, 有机体更倾向于选择电子转移效率高的生物过程, 而每还原 $1 \mathrm{~mol}$ 的 $\mathrm{NO}_{3}^{-}-\mathrm{N}$, DNRA 过程 
转移 8 个电子, DNF 过程只转移 5 个电子这可能是导致巢湖重污染河流湿地和重污染湖泊湿地沉积物 - 水 界面具有较高的 DNRA 速率的原因. 除了以上环境因素引起的原因外, 根据 $D_{14} / D_{15}$ 算出的 DNRA 实际速率 $\left(R_{\mathrm{DNRA}}^{14}\right)$ 可能与真实速率存在一定的偏差; 在微生物吸收硝态氮进行同化作用的过程中, 微生物合成的氨氮 可能会再次释放到水体中 (ANRA 过程), 该过程的存在可能会导致对 DNRA 速率的高估.

表 3 湖泊、水库等湿地反硝化、DNRA 速率以及 DNRA 在硝酸盐异养还原过程中所占的比重

Tab.3 Denitrification and DNRA rates and the proportion of the DNRA in the total dissimilatory nitrate reduction process in lake wetland and reservoir wetland

\begin{tabular}{|c|c|c|c|c|c|}
\hline 沉积物类型 & 地点 & $\begin{array}{c}R_{\mathrm{DNF}} / \\
\left(\mu \mathrm{mol} /\left(\mathrm{m}^{2} \cdot \mathrm{h}\right)\right)\end{array}$ & $\begin{array}{c}R_{\mathrm{DNRA}}{ }^{\prime} \\
\left(\mu \mathrm{mol} /\left(\mathrm{m}^{2} \cdot \mathrm{h}\right)\right)\end{array}$ & $\frac{R_{\mathrm{DNRA}}}{R_{\mathrm{DNRA}}+R_{\mathrm{DNF}}}$ & $\begin{array}{l}\text { 计算 } \\
\text { 类别 }\end{array}$ \\
\hline 淡水湖泊湿地 & $\begin{array}{l}\text { Waco 湿地 }{ }^{[4]} \\
\text { (美国西部) }\end{array}$ & $54 \sim 278$ & $1.3 \sim 33$ & $5 \%$ & $\dagger$ \\
\hline 中度营养化湖泊 & $\begin{array}{l}\text { Ca'stanga 湖 }[10] \\
\text { (意大利北部) }\end{array}$ & $(138 \pm 11) \sim(171 \pm 27)$ & $1 \sim 9$ & $2 \%$ & $\ddagger$ \\
\hline 重度富营养化湖泊 & $\begin{array}{l}\text { Lago Verde 湖 [10] } \\
\text { (意大利北部) }\end{array}$ & $(31 \pm 8) \sim(125 \pm 22)$ & $1 \sim 9$ & $2 \%$ & $\ddagger$ \\
\hline 重度富营养化湖泊 & $\begin{array}{c}\text { Slaen 湖 } \\
\text { ( 丹麦 })\end{array}$ & 33 & $3.4 \sim 14.1$ & $20 \% \sim 33 \%$ & $\dagger$ \\
\hline 富营养化湖泊 & $\begin{array}{c}\text { Keurusselkä 湖 } \\
\text { (芬兰西部) }\end{array}$ & $6.6 \sim 245.4$ & $0.6 \sim 254.5$ & $9 \% \sim 52 \%$ & $\ddagger$ \\
\hline 富营养化水库 & $\begin{array}{c}\text { Rzeszów 水库 }{ }^{[20]} \\
\text { (波兰东南部) }\end{array}$ & 220.7 & 11.3 & $4.87 \%$ & $\ddagger$ \\
\hline 富营养化湖泊 & $\begin{array}{l}\text { Baldegg 湖 }{ }^{[25]} \\
\quad(\text { 瑞士 })\end{array}$ & 179.17 & 8.33 & $4.44 \%$ & $\ddagger$ \\
\hline 水库 & $\begin{array}{c}\text { Dayton 市水库 }{ }^{[14]} \\
\text { (美国中西部) }\end{array}$ & $\begin{array}{l}2.8 \pm 0.3^{\mathrm{a}} \\
11.2 \pm 1.9^{\mathrm{b}}\end{array}$ & $\begin{array}{c}2.8 \pm 0.6^{\mathrm{a}} \\
53.9 \pm 14.7^{\mathrm{b}}\end{array}$ & $\begin{array}{l}50.4 \% \pm 8.1 \%^{\mathrm{a}} \\
18.7 \% \pm 4.1 \%^{\mathrm{b}}\end{array}$ & $\dagger$ \\
\hline 富营养化水库 & Solina 水库 ${ }^{[24]}$ & 67.725 & 6.425 & $8.66 \%$ & $\ddagger$ \\
\hline 本实验 & 重污染河流湿地 & 99.98 & 79.82 & $44.4 \%$ & \\
\hline 本实验 & 重污染湖泊湿地 & 63.67 & 63.05 & $49.8 \%$ & $\ddagger$ \\
\hline 本实验 & 碟形湖湿地 & 6.36 & 0.51 & $7.4 \%$ & \\
\hline
\end{tabular}

$\dagger:$ 潜在 DNF 和 DNRA 速率; $¥:$ 实际 DNF 和 DNRA 速率. $\mathrm{a}$ : 对照组, 未添加底栖动物培养实验 $; \mathrm{b}$ : 实验组, 底栖动物添加培 养实验.

许多研究表明, 硝态氮浓度、有机碳浓度和可利用性、硫化物、温度、氧化还原条件等均会对 DNRA 速率 产生影响 ${ }^{[30-32]}$. 在本研究中, DNRA 过程是非常重要的硝态氮异养还原过程, 实验测得的 DNRA 速率约占总 的硝态氮异养还原过程 (DNRA+DNF) 的 7\% 49\% , 且不同类型湿地间存在很大差异. 巢湖重污染河流、湖 泊湿地沉积物 DNRA 速率在硝态氮异养还原过程中所占的比重分别为 $44 \%$ 和 $49 \%$, 而碟形湖湿地中 DNRA 在异养还原过程中所占的比重仅为 7\%. 从这种差异中可以看出, 重污染河流湿地和重污染湖泊湿地较高的 硝酸盐负荷、较高的的有机质含量等环境条件不仅有利于 DNRA 过程的进行, 而且有利于 DNRA 过程对 DNF 过程的竞争, 使得 DNRA 速率以及 DNRA 比重相较于碟形湖湿地均显著升高. 由于 DNRA 和 DNF 均是 以硝酸盐作为底物的还原过程, 因此, 存在着底物竞争作用, 这种竞争作用影响了硝酸盐的归趋方式, 过高 的 DNRA 速率不利于水体氮负荷的去除和湿地净化功能的发挥.

\section{4 结论}

1) 重污染河流湿地、湖泊湿地与碟形湖湿地沉积物一水界面的营养盐通量具有较大差异, 重污染河流、 湖泊湿地沉积物均表现为 $\mathrm{NH}_{4}^{+}-\mathrm{N}$ 的源、 $\mathrm{NO}_{3}^{-}-\mathrm{N}$ 的汇; 而碟形湖湿地则表现为 $\mathrm{NH}_{4}^{+}-\mathrm{N}$ 的汇、 $\mathrm{NO}_{3}^{-}-\mathrm{N}$ 的源. 但在 3 种类型湿地沉积物中 DIN 总体上均表现为从上覆水迁移到沉积物中. 
2) 在不同类型湿地中 $\mathrm{NO}_{3}^{-}-\mathrm{N}$ 浓度是控制反硝化速率和反硝化方式的主要因素, $\mathrm{NO}_{3}^{-}-\mathrm{N}$ 浓度与反硝化速 率和 $D_{\mathrm{w}}$ 在反硝化中所占的比重均呈现正相关.

3) DNF 速率和 DNRA 速率均表现为重污染河流湿地>重污染湖泊湿地>碟形湖湿地, 且巢湖重污染湿 地 DNRA 速率在 $\mathrm{NO}_{3}^{-}-\mathrm{N}$ 异养还原过程中所占的比重也明显高于碟形湖湿地, 因此推测较高的硝酸盐负荷、 较高的有机质含量不仅有利于 DNRA 过程的进行,而且有利于 DNRA 过程对 DNF 过程的竞争. 致谢:感谢鄱阳湖湖泊湿地观测研究站对本文野外样品采集提供的帮助.

\section{5 参考文献}

[ 1 ] Tobias C, Giblin A, McClelland J et al. Sediment DIN fluxes and preferential recycling of benthic microalgal nitrogen in a shallow macrotidal estuary. Marine Ecology Progress Series, 2003, 257: 25-36. DOI: 10.3354/meps257025.

[ 2 ] Xu Hui, Zhang Lu, Shang Jingge et al. Denitrification and anammox on the sediment-water interface in the Meiliang Bay of Lake Taihu. J Lake Sci, 2009, 21(6) : 775-781 (in Chinese with English abstract). DOI: 10.18307/2009.0604. [ 徐徽, 张路, 商景阁等. 太湖梅梁湾水土界面反硝化和厌氧氨氧化. 湖泊科学, 2009, 21(6): 775-781.]

[ 3 ] Turner RE, Rabalais NN. Linking landscape and water quality in the Mississippi River basin for 200 years. BioScience, $2003, \mathbf{5 3}(6)$ : 563-572. DOI: 10.1641/0006-3568(2003)053[0563: LLAWQI] 2. 0. CO;2.

[ 4 ] Scott JT, McCarthy MJ, Gardner WS et al. Denitrification, dissimilatory nitrate reduction to ammonium, and nitrogen fixation along a nitrate concentration gradient in a created freshwater wetland. Biogeochemistry, 2008, 87(1) : 99-111. DOI: 10.1007/s10533-007-9171-6.

[ 5 ] Burgin AJ, Hamilton SK. Have we overemphasized the role of denitrification in aquatic ecosystems? A review of nitrate removal pathways. Frontiers in Ecology and the Environment, 2007, 5(2) : 89-96. DOI: 10.1890/1540-9295(2007) 5[89: HWOTRO $] 2.0 . \mathrm{CO} ; 2$.

[ 6 ] Howarth RW, Marino R. Nitrogen as the limiting nutrient for eutrophication in coastal marine ecosystems: evolving views over three decades. Limnology and Oceanography, 2006, 51 (1part2): 364-376. DOI: 10.4319/lo. 2006.51.1_part_ 2.0364 .

[ 7 ] Giblin AE, Tobias CR, Song B et al. The importance of dissimilatory nitrate reduction to ammonium (DNRA) in the nitrogen cycle of coastal ecosystems. Oceanography, 2013, 26(3) : 124-131. DOI : 10.5670/oceanog.2013.54.

[ 8 ] Song Guodong. Benthic nitrogen loss and transformations in the east china sea[Dissertation]. Qingdao: Ocean University of China, 2013 (in Chinese with English abstract). [ 宋国栋. 东海沉积物中氮循环的关键过程 [学位论文]. 青岛: 中国 海洋大学, 2013.]

[ 9 ] Howarth RW, Billen G, Swaney D. Regional nitrogen budgets and riverine N and P fluxes for the drainages to the North Atlantic Ocean: natural and human influences. Oceanographic Literature Review, 1997, 44(5) : 448. DOI: 10.1007/97894-009-1776-7_3.

[10] Nizzoli D, Carraro E, Nigro V et al. Effect of organic enrichment and thermal regime on denitrification and dissimilatory nitrate reduction to ammonium (DNRA) in hypolimnetic sediments of two lowland lakes. Water Research, 2010, 44(9) : 2715-2724. DOI: 10.1016/j.watres.2010.02.002.

[11] Kelly-Gerreyn BA, Trimmer M, Hydes DJ. A diagenetic model discriminating denitrification and dissimilatory nitrate reduction to ammonium in a temperate estuarine sediment. Marine Ecology Progress Series, 2001, 220: 33-46. DOI: 10. 3354/meps220033.

[12] An S, Gardner WS. Dissimilatory nitrate reduction to ammonium (DNRA) as a nitrogen link, versus denitrification as a sink in a shallow estuary (Laguna Madre/Baffin Bay, Texas). Marine Ecology Progress Series, 2002, 237(1499) : 41-50. DOI : $10.3354 /$ meps 237041 .

[13] Yin SX, Chen D, Chen LM et al. Dissimilatory nitrate reduction to ammonium and responsible microorganisms in two Chinese and Australian paddy soils. Soil Biology and Biochemistry, 2002, 34( 8 ) : 1131-1137. DOI : 10.1016/S0038-0717 ( 02 ) 00049-4.

[14] Nogaro G, Burgin AJ. Influence of bioturbation on denitrification and dissimilatory nitrate reduction to ammonium (DNRA) in freshwater sediments. Biogeochemistry, 2014, 120(1/2/3) : 279-294. DOI: 10.1007/s10533-014-9995-9.

[15] Liang Yue. Biogeochemistry of heavy metal and nitrogen and isotopic tracing in Banghu (Lake Poyang) [Dissertation]. 
Nanchang: Nanchang University, 2014(in Chinese with English abstract). [ 梁越. 鄱阳湖区蚌湖重金属及氮的生物地 球化学与同位素示踪 [学位论文]. 南昌: 南昌大学, 2014.]

[16] Wang Shuhang, Jiang Xia, Jin Xiangcan. Classification and pollution characteristic analysis for inflow rivers of Lake Chaohu. Chinese Journal of Environmental Science, 2011, 32(10): 2834-2839( in Chinese with English abstract). [王书航, 姜霞, 金相灿. 巢湖人湖河流分类及污染特征分析. 环境科学, 2011, 32(10): 2834-2839.]

[17] Yin G, Hou L, Liu M et al. A novel membrane inlet mass spectrometer method to measure ${ }^{15} \mathrm{NH}_{4}^{+}$for isotope-enrichment experiments in aquatic ecosystems. Environmental Science \& Technology, 2014, 48 ( 16 ) : 9555-9562. DOI: 10. $1021 /$ es501261s.

[18] Kunu TM, Sullivan MB, Cornwell JC et al. Denitrification in estuarine sediments determined by membrane inlet mass spectrometry. Limnology and Oceanography, 1998, 43(2) : 334-339. DOI: 10.4319/lo.1998.43.2.0334.

[19] Nielsen LP. Denitrification in sediment determined from nitrogen isotope pairing. FEMS Microbiology Letters, 1992,86 (4) : 357-362. DOI: 10.1111/j.1574-6941.1992.tb01771.x.

[20] Steingruber SM, Friedrich J, Gächter R et al. Measurement of denitrification in sediments with the ${ }^{15} \mathrm{~N}$ isotope pairing technique. Applied and Environmental Microbiology, 2001, 67 (9): 3771-3778. DOI: 10. 1128/AEM. 67. 9. 37713778.2001 .

[21] Hou L, Liu M, Carini SA et al. Transformation and fate of nitrate near the sediment-water interface of Copano Bay. Continental Shelf Research, 2012, 35: 86-94. DOI: 10.1016/j.csr.2012.01.004.

[22] Gruca-Rokosz R, Tomaszek JA, Koszelnik P. Competitiveness of dissimilatory nitrate reduction processes in bottom sediment of Rzeszów reservoir. Environment Protection Engineering, 2009, 35(2) : 5-13. DOI : 10.1007/BF0034553.

[23] Li Qiang, Huo Shouliang, Wang Xiaowei et al. Distribution and correlation of nutrients and particle size in surface sediments of Lake Chaohu and its inflow rivers. Journal of Environmental Engineering Technology, 2013, 3(2) : 147-155(in Chinese with English abstract). [ 李强, 霍守亮, 王晓伟等. 巢湖及其人湖河流表层沉积物营养盐和粒度的分布及其 关系研究. 环境工程技术学报, 2013, 3(2): 147-155.]

[24] Yang Chunxia. The effect of organic matter and submerged macrophyte on nitrogen and phosphorus mineralization and pools in the lake sediment[Dissertation]. Beijing: Chinese Research Academy of Environmental Sciences, 2009 (in Chinese with English abstract). [ 杨春霞. 有机质及沉水植物对湖泊沉积物界面氮磷矿化与赋存的影响 [学位论文]. 北京: 中国环境科学研究院, 2009.]

[25] Pelegrí SP, Blackburn TH. Nitrogen cycling in lake sediments bioturbated by Chironomus plumosus larvae, under different degrees of oxygenation. Hydrobiologia, 1996, 325(3) : 231-238. DOI : 10.1007/BF00014989.

[26] Hasari M. Denitrification and dissimilatory nitrate reduction to ammonium in a lake receiving wastewater effluent[Dissertation]. University of Jyväskylä, Faculty of Science, 2015.

[27] Tomaszek JA, Rokosz GR. Rates of dissimilatory nitrate reduction to ammonium in two polish reservoirs: Impacts of temperature, organic matter content, and nitrate concentration. Environmental Technology, 2007, 28(7) : 771-778. DOI: 10. 1080/09593332808618834.

[28] Mengis M, Gächter R, Wehrli B et al. Nitrogen elimination in two deep eutrophic lakes. Limnology and Oceanography, 1997, 42(7) : 1530-1543. DOI: 10.4319/lo.1997.42.7.1530.

[29] Dong LF, Sobey MN, Smith C et al. Dissimilatory reduction of nitrate to ammonium (DNRA) not denitrification or anammox dominates benthic nitrate reduction in tropical estuaries. Limnology and Oceanography, 2011, 56: 279-291. DOI: 10. 4319/lo.2011.56.1.0279.

[30 ] Burgin $\mathrm{AJ}$, Hamilton $\mathrm{SK} . \mathrm{NO}_{3}^{-}$driven $\mathrm{SO}_{4}^{2-}$ production in freshwater ecosystems: Implications for $\mathrm{N}$ and $\mathrm{S}$ cycling. Ecosystems, 2008, 11(6) : 908-922. DOI : 10.1007/s10021-008-9169-5.

[31] Tiedje JM. Biology of anaerobic microorganisms. New York: John Wiley and Sons Inc, 1988: 179-244.

[32] Patricia B, Patrick O, Alain C. Simultaneous occurrence of denitrification and nitrate ammonification in sediments of the French Mediterranean Coast. Hydrobiologia, 1998, 389(1/2/3): 169-182. DOI : 10.1023/A 1003585115481. 